\title{
Application of Fractional Fourier Transform to Moving Target Indication via Along-Track Interferometry
}

\author{
Shen Chiu \\ Radar System Section, Defence R\&D Canada (DRDC), 3701 Carling Avenue, Ottawa, ON, Canada K1A 0Z4
}

Email: shen.chiu@drdc-rddc.gc.ca

Received 21 June 2004; Revised 4 March 2005

\begin{abstract}
A relatively unknown yet powerful technique, the so-called fractional Fourier transform (FrFT), is applied to SAR along-track interferometry (SAR-ATI) in order to estimate moving target parameters. By mapping a target's signal onto a fractional Fourier axis, the FrFT permits a constant-velocity target to be focused in the fractional Fourier domain thereby affording orders of magnitude improvement in SCR. Moving target velocity and position parameters are derived and expressed in terms of an optimum fractional angle $\alpha$ and a measured fractional Fourier position $u_{p}$, allowing a target to be accurately repositioned and its velocity components computed without actually forming an SAR image. The new estimation algorithm is compared with the matched filter bank approach, showing some of the advantages of the FrFT method. The proposed technique is applied to the data acquired by the two-aperture CV580 airborne radar system configured in its along-track mode. Results show that the method is effective in estimating target velocity and position parameters.
\end{abstract}

Keywords and phrases: fractional Fourier transform, along-track interferometry, parameter estimation.

\section{INTRODUCTION}

Canada's RADARSAT-2 commercial SAR satellite, to be launched in spring 2006, will have an experimental mode (called MODEX for moving object detection experiment) that will allow the full antenna to be broken into two subapertures with two parallel receivers to define two independent data channels [1]. These two subapertures, arranged to lie along the flight path, record two echoes (the dual-receive mode), one from each wing for every pulse transmitted at the full antenna. The two apertures enable one to detect targets with nonzero radial velocity by providing essentially two identical views of the observed scene but at slightly different times. In addition to the dual-receive mode of operation, RADARSAT-2 will also support an alternating-transmit mode where pulses are transmitted alternately from each wing and received alternately on each wing. This mode allows greater separation of the two-way phase centers in the along-track direction and also the possibility of generating a third phase center for three-aperture ground moving target indication (GMTI). This mode of operation is currently being investigated in preparation for RADARSAT-2 MODEX demonstration but is not examined in this paper, since it has been recognized that a two-aperture approach is suboptimum $[2,3]$.

It can be shown that a moving target with a slant range velocity $v_{r}$ causes a differential phase shift $\varphi_{\text {ATI }}=4 \pi v_{r} \tau / \lambda$
( $\tau$ is the time between two observations and $\lambda$ is wavelength), which may be detected by interferometric combination of the signals from a two-channel along-track SAR system [4]. Moving target signals are embedded in the imaged stationary scene, which is called "clutter." The interferometric phase $\varphi_{\text {ATI }}$ is often used to estimate a target's radial velocity and azimuth shift, without considering the fact that $\varphi_{\text {ATI }}$ is corrupted by the overlapping stationary clutter (cf. [5]). This may lead to serious errors in velocity and position estimates. In order to accurately estimate target's true position and velocity, clutter contamination of the target's signal must be minimized. For a two-aperture radar system, the additional degree of freedom provided by the second aperture can be used to cancel clutter via the subtractive displaced phase center antenna (DPCA), providing GMTI information. However, the expended degree of freedom can no longer be used to estimate target parameters. The ATI, on the other hand, contains target parameter information but is contaminated by scene clutter because the ATI method does not actually cancel clutter in the sense of removing clutter from the target. It only nulls the clutter's interferometric phase but does not truly cancel the clutter as does the DPCA.

The fractional Fourier transform (FrFT) is a relatively unknown yet powerful technique, which when used in combination with the ATI, provides significant signal-to-clutter ratio (SCR) enhancement and at the same time allows target position and velocity parameters to be accurately estimated. 


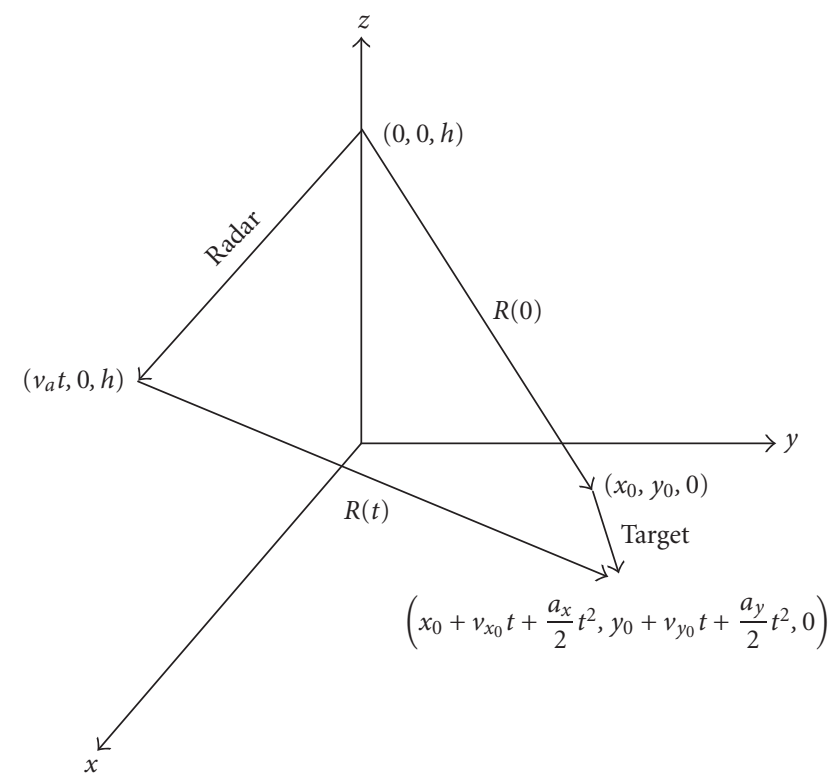

FIgURE 1: A target moving in a flat-earth geometry with motion components in $x$ and $y$ direction. The radar moves in a positive $x$ direction.

The FrFT is a generalization of the regular Fourier transform (FT) in that the FT transforms a signal from time domain to frequency domain, the FrFT transforms it into a fractional Fourier domain, which is a hybridized time-frequency domain. A constant-velocity target signal, which is a linear chirp, can be focused in an optimum fractional Fourier domain. Moving target position and motion parameters can be derived and expressed in terms of a best FrFT angle $\alpha$ and position $u_{p}$, which can be computed from the experimentally measured data.

\section{THEORY}

\subsection{Moving target signal model}

The phase history of a moving target in a flat-earth geometry, as shown in Figure 1, can be modelled as

$$
\begin{aligned}
& \varphi(t) \\
& =-2 k R(t) \\
& =-2 k \sqrt{\left[x_{0}+\left(v_{x_{0}}-v_{a}\right) t+\frac{a_{x}}{2} t^{2}\right]^{2}+\left[y_{0}+v_{y_{0}} t+\frac{a_{y}}{2} t^{2}\right]^{2}+h^{2},}
\end{aligned}
$$

where $R(t)$ is the range history of the moving target, $k=$ $2 \pi / \lambda$, and $v$ and $a$ are target velocity and acceleration, respectively. Subscript " 0 " denotes velocity at time zero, and $x$ and $y$ are along-track and across-track components, respectively. The target velocity and position at broadside time $t_{b}$

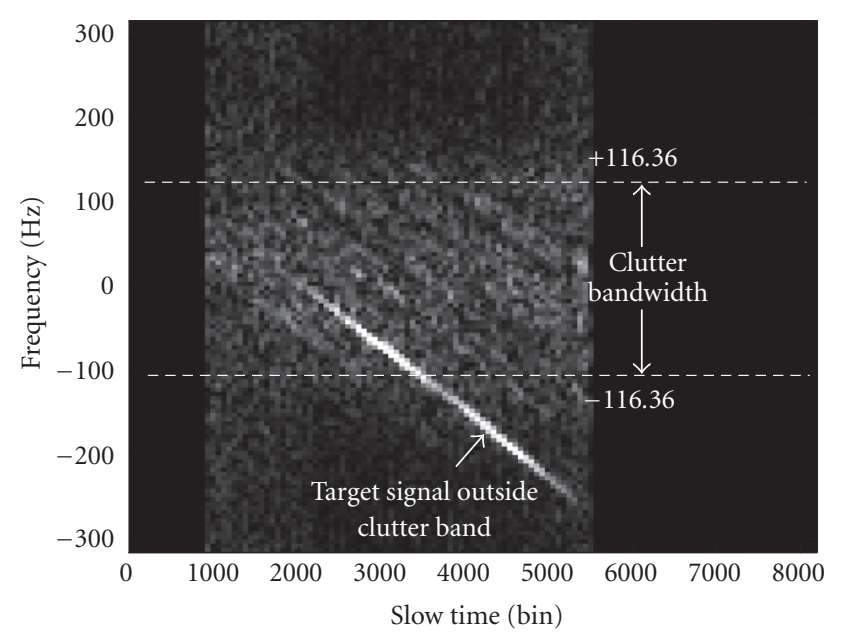

FIgURE 2: Time-frequency distribution of target T8 obtained via short-time Fourier transform, showing the Doppler-shifted linear chirp of the target overlaid on stationary background clutter $(3 \mathrm{~dB}$ beamwidth is also shown).

are therefore

$$
\begin{aligned}
& v_{x b}=v_{x_{0}}+a_{x} t_{b}, \\
& v_{y b}=v_{y_{0}}+a_{y} t_{b}, \\
& x_{b}=v_{a} t_{b}=x_{0}+v_{x_{0}} t_{b}+\frac{a_{x} t_{b}^{2}}{2}, \\
& y_{b}=y_{0}+v_{y_{0}} t_{b}+\frac{a_{y} t_{b}^{2}}{2} .
\end{aligned}
$$

The Taylor expansion of (1) about broadside time $t_{b}$ can be written as

$$
\begin{aligned}
\varphi(t) \approx-2 k\{ & R_{b}+v_{y b} \gamma\left(t-t_{b}\right)+\frac{1}{2 R_{b}}\left(v_{\mathrm{rel}}^{2}+y_{b} a_{y}\right)\left(t-t_{b}\right)^{2} \\
& \left.+\frac{1}{2 R_{b}}\left[\left(v_{x b}-v_{a}\right) a_{x}+v_{y b} a_{y}\left(1-\gamma^{2}\right)\right]\left(t-t_{b}\right)^{3}+\cdots\right\},
\end{aligned}
$$

where $R_{b}$ is target's slant range at broadside and

$$
\begin{gathered}
\gamma=\frac{y_{b}}{R_{b}} \\
v_{\text {rel }}^{2}=\left(v_{x b}-v_{a}\right)^{2}+v_{y b}^{2}\left(1-\frac{y_{b}^{2}}{R_{b}^{2}}\right) .
\end{gathered}
$$

The signal of a moving target in "slow time" can, therefore, be modelled as

$$
s(t)=\operatorname{rect}\left[\frac{t-t_{b}}{T}\right] e^{j \varphi(t)},
$$

which is a finite-time linear chirp with duration $T$ centered at time $t=t_{b}$. In the real signal, the "rect" function is also modulated by the antenna pattern.

Figure 2 shows the time-frequency (TF) plot, obtained via the short-time Fourier transform (STFT) technique 
[6], of a moving target's range-compressed signal (T8; see Figure 8) superimposed on a stationary residual clutter. The target's range-compressed signal is a linear chirp in slow time, which appears as a slanted line in the TF plane. Because of the target's across-track velocity component $v_{y b}$ (i.e., the first-order term in the Taylor expansion), the signal is shifted in frequency (in this case in the negative direction). If the target has non-negligible along-track and/or across-track velocity components ( $v_{x b}$ and $v_{y b}$ ) with respect to platform velocity $v_{a}$ and/or non-negligible along-track acceleration $a_{x}$ (i.e., the second order-terms in the Taylor expansion or the slope of the chirp), then its signal will be rotated slightly in the TF-plane with respect to a stationary point target. The signal remains a linear chirp as long as the target has no acceleration components, $a_{x}$ and $a_{y}$, or other higher-order terms.

\subsection{Fractional Fourier transform}

The fractional Fourier transform with rotational angle $\alpha$ of a signal $f(t)$ is defined as $[7,8]$

$$
F_{\alpha}(u)=\mathfrak{R}^{\alpha}[f](u)=\int_{-\infty}^{\infty} f(t) K_{\alpha}(t, u) d t
$$

where for $\alpha$ not equal to zero or a multiple of $\pi$, the kernel $K_{\alpha}(t, u)$ is given by

$$
\begin{aligned}
K_{\alpha}(t, u) & =c e^{j 2 \pi a\left[t^{2}+u^{2}-2 b u t\right]}, \\
a=\frac{\cot \alpha}{2}, \quad b & =\sec \alpha, \quad c=\sqrt{1-j \cot \alpha} .
\end{aligned}
$$

The FrFT with parameter $\alpha$ can be considered as a generalization of the conventional Fourier transform (FT). Thus, the FrFT for $\alpha=\pi / 2$ and $\alpha=-\pi / 2$ reduces to the conventional and inverse FT, respectively. Multiplying both sides of (6) by $e^{-j 2 \pi a u^{2}}$, one obtains

$$
e^{-j 2 \pi a u^{2}} F_{\alpha}(u)=\widetilde{F}_{\alpha}(u)=c \int_{-\infty}^{\infty} f(t) e^{j 2 \pi a\left(t^{2}-2 b u t\right)} d t .
$$

Substituting $v / 2 a b$ for $u$ in (8), it becomes

$$
\begin{aligned}
\widetilde{F}_{\alpha}\left(\frac{v}{2 a b}\right) & =c \int_{-\infty}^{\infty} f(t) e^{j 2 \pi\left(a t^{2}-\gamma t\right)} d t \\
& =\int_{-\infty}^{\infty}\left\{c f(t) e^{j 2 \pi a t^{2}}\right\} e^{-j 2 \pi v t} d t \\
& =\int_{-\infty}^{\infty} g_{\alpha}(t) e^{-j 2 \pi v t} d t \\
& =G_{\alpha}(\nu)
\end{aligned}
$$

where $g_{\alpha}(t)=c f(t) \exp \left(j 2 \pi a t^{2}\right)$. The second last line in (9) shows that the FrFT is a variation of the standard FT. As such, many of its properties, such as its inverse formula and sampling theorems for band-limited and time-limited signals, can be easily derived from those of the FT by a simple change of variable. With respect to the parameter $\alpha$, the FrFT is continuous, periodic ( $\mathfrak{R}^{\alpha}=\mathfrak{R}^{\alpha+2 \pi n}$, with $n$ an integer), and additive $\left(\mathfrak{R}^{\alpha} \mathfrak{R}^{\beta}=\mathfrak{R}^{\alpha+\beta}\right)$, and has the symmetry relation $\mathfrak{R}^{\alpha}\left[f^{*}\right](u)=\left\{\mathfrak{R}^{-\alpha}[f](u)\right\}^{*}$. The inverse FrFT can thus be written as

$$
f(t)=\mathfrak{R}^{-\alpha}\left[F_{\alpha}\right](t)=\int_{-\infty}^{\infty} F_{\alpha}(u) K_{-\alpha}(t, u) d u .
$$

The FrFT maps the signal onto a rotated (or fractional) frequency axis such that the originally slanted chirp energy becomes perpendicular to the rotated axis and, thus, highly compressed or focused as illustrated in Figure 3. Also shown in Figure 3 (insert) is a "measured" moving target (T10) mapped onto the optimum fractional frequency axis. The target energy, as can be seen, is highly compressed. Note that the clutter has been suppressed by subtracting the aft channel from the fore channel after time delay compensation (or coregistration) [9]. The observed residual clutter can be attributed to scene decorrelation, possibly caused by scene motions and/or by slightly different observation angles, during the fore and aft measurements. The residual clutter (plus noise) surrounding the signal chirp can be filtered out if desired. The signal strength is dramatically increased due to pulse compression, resulting in a significant improvement in the signal-to-clutter ratio (SCR) and, thus, in the interferometric phase estimation accuracy [10].

The pulse compression via the FrFT is more or less equivalent to SAR azimuth processing via a matched filter (MF) that matches to the coefficient of the second-order term (i.e., the slope of the chirp) in (3). The signal compression via the FrFT is not dependent on the first-order term (i.e., the Doppler shift) but only on second- and higher-order terms in the Taylor expansion, in contrast to the matched filter approach. In the limiting case of small observation angular interval, the FrFT method will lead to a focused target if the target is moving at a constant velocity throughout the synthetic aperture time or only slightly accelerating in the across-track direction. Unlike a matched filter, which is capable of fully "matching" to the target's motion if the motion parameters are known, the FrFT cannot, in principle, fully focus a target that is accelerating in the along-track direction (i.e., $a_{x} \neq 0$ ) because $a_{x}$ introduces nonlinearity in the signal chirp. The across-track acceleration, on the other hand, introduces mainly a Doppler-rate (or slope) change in the chirp and, therefore, does not lead to significant nonlinearity $[4,11]$. The ability of a matched filter to focus an accelerating target does not necessarily mean that the target's parameters can be estimated more easily than the FrFT approach. As it turns out (to be shown in Section 3.3), the obvious advantage of the FrFT method is that it has fewer intermediate parameters to be estimated than the MF approach in the case of constant velocity targets, making the FrFT method a more robust and preferred technique. 


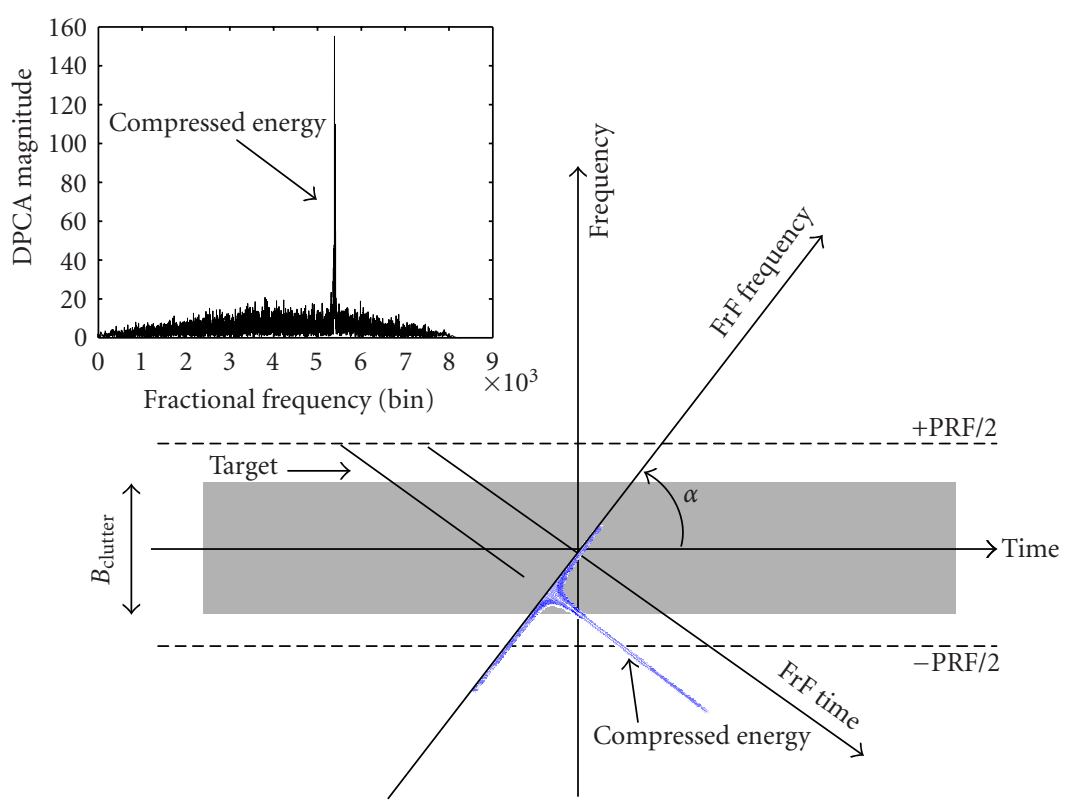

FIGURE 3: Illustration of a target energy focused via the FrFT. The insert shows an actual moving target's (T10) signal compressed in the fractional frequency domain.

This paper will not consider acceleration effects further, and from now on the subscripts $b$ and 0 will be dropped from the velocity terms.

\section{PARAMETER ESTIMATION ALGORITHMS}

In this section we will describe parameter estimation algorithms based on the FrFT and then compare them to the MF approach. The comparison addresses only the relative ease of the two methods in accurately estimating the ATI differential phase $\varphi_{\mathrm{ATI}}$, which is the key intermediate parameter for determining the target velocity components.

\subsection{Negligible along-track velocity}

Having detected the targets and extracted their tracks or range histories (see Section 4 and Figure 8) from the rangecompressed but azimuth-uncompressed fore and aft data pair, each target is individually analyzed via the following parameter estimation algorithm. The FrFT is first applied to each target to maximize its SCR by mapping its energy onto a fractional Fourier axis. This is done by scanning through the angular parameter $\alpha$ in (6) to maximize the SCR. Then the ATI differential phase is computed for this optimum fractional angle $\alpha$ to get the "best" (or the least contaminated) $\varphi_{\text {ATI }}$. The radial velocity $v_{r}$ at the broadside (also equal to the slant range velocity) is estimated from the interferometric phase $\varphi_{\text {ATI }}$ using the relationship [12]

$$
\varphi_{\mathrm{ATI}}=\frac{4 \pi \tau v_{r}}{\lambda}=\frac{k d v_{y} \gamma}{v_{a}}
$$

where $\tau$ is the time delay between the pair of received signals and $d$ is the distance between the centers of two subapertures. The ground range velocity $v_{y}$ is calculated from $v_{y}=v_{r} / \sin \eta$, where $\eta$ is the angle of incidence and $\gamma=$ $\sin \eta=y_{b} / R_{b}$. The along-track velocity $v_{x}$, on the other hand, is estimated from the fractional (or rotational) angle $\alpha$, which can be obtained when the target is best focused in the fractional Fourier domain, that is, by setting (6) equal to a "sinc" function:

$$
\begin{aligned}
F_{\alpha}(u) & =\int_{-\infty}^{\infty} \operatorname{rect}\left(\frac{t-t_{b}}{T}\right) e^{j \varphi(t)} K_{\alpha}(t, u) d t \\
& =\int_{t_{b}-T / 2}^{t_{b}+T / 2} e^{j \varphi(t)} K_{\alpha}(t, u) d t \\
& =\kappa \operatorname{sinc}\left[\mu\left(u-u_{p}\right)\right] e^{j \varphi^{\prime}(u)},
\end{aligned}
$$

where $\kappa$ and $\mu$ are constants, $T$ is signal length centered at broadside time $t_{b}, \varphi^{\prime}(u)$ is signal's phase in fractional Fourier domain, and $u_{p}$ is the target position on the fractional Fourier axis, which can be shown to be equal to equation

$$
u_{p}=\frac{1}{4 \pi a b}\left(-2 k \gamma v_{y}+\frac{2 k v_{\mathrm{rel}}^{2} t_{b}}{R_{b}}\right)
$$

Note that (12) is equal to a sinc function only when the $t^{2}$ term in the exponential of the integrand is equal to zero, thus

$$
\alpha=\operatorname{acot}\left\{\frac{2 N}{R_{b} \lambda f_{s}^{2}}\left[\left(v_{x}-v_{a}\right)^{2}+v_{y}^{2}\left(1-\gamma^{2}\right)\right]\right\}
$$


or

$$
v_{x}=v_{a}-\sqrt{\frac{R_{b} \lambda f_{s}^{2} \cot \alpha}{2 N}-v_{y}^{2}\left(1-\gamma^{2}\right)},
$$

where $N$ is sample length and $f_{s}$ is sampling (or pulse repetition) frequency. The $f_{s}^{2} / N$ factor is introduced to convert time-frequency units to dimensionless time-frequency samples in the digitized FrFT domain. The target azimuth shift correction $\Delta x$ can be derived by setting the derivative of phase history (3) to zero and solving for $\left(t_{c}-t_{b}\right)$, which yields the azimuth shift correction, $\Delta x=v_{a}\left(t_{c}-t_{b}\right)$,

$$
\Delta x=\frac{v_{y} v_{a} y_{b}}{\left(v_{x}-v_{a}\right)^{2}+v_{y}^{2}\left(1-\gamma^{2}\right)} \approx \frac{v_{y} y_{b}}{v_{a}}=\frac{v_{r} R_{b}}{v_{a}},
$$

where the approximation $(\approx)$ is valid only when $v_{a} \gg v_{x}$. In order to calculate $t_{b}$ from (16), one still needs to know $t_{c}$, the time of closest approach. An alternate and better method to estimate $t_{b}$ is via the FrFT, that is solving (13) for $t_{b}$ :

$$
t_{b}=\frac{R_{b}}{k v_{\mathrm{rel}}^{2}}\left(\frac{\pi u_{p}}{\sin \alpha}+k \gamma v_{y}\right)
$$

Expressing in dimensionless time and frequency samples (instead of units of second and Hertz), (17) becomes

$$
t_{b}=\left(\frac{f_{s}^{2}}{N}\right) \frac{R_{b}}{v_{\text {rel }}^{2}}\left[\frac{\pi u_{p}}{k \sin \alpha}+\left(\frac{N}{f_{s}}\right) \gamma v_{y}\right]
$$

Equation (18) can also be derived from the geometry of the signal chirp in frequency-time plane as shown in Figure 4. From the figure, the time of closest approach $t_{c}$ is equal to

$$
t_{c}=\frac{u_{p}}{\cos \alpha}
$$

and " $\tan \alpha$ " is equal to

$$
\tan \alpha=\frac{t_{c}-t_{b}}{f_{D}}
$$

Combining (19) and (20) gives

$$
t_{b}=\tan \alpha\left(\frac{u_{p}}{\sin \alpha}-f_{D}\right)
$$

which can be shown to be equal to (18) when substituting for

$$
\begin{aligned}
f_{D} & =-\frac{N k v_{r}}{f_{s} \pi}=-\frac{N k v_{y} \gamma}{f_{s} \pi}, \\
\tan \alpha & =\frac{f_{s}^{2} R_{b} \lambda}{2 N v_{\text {rel }}^{2}}=\frac{f_{s}^{2} R_{b} \pi}{N v_{\text {rel }}^{2} k} .
\end{aligned}
$$

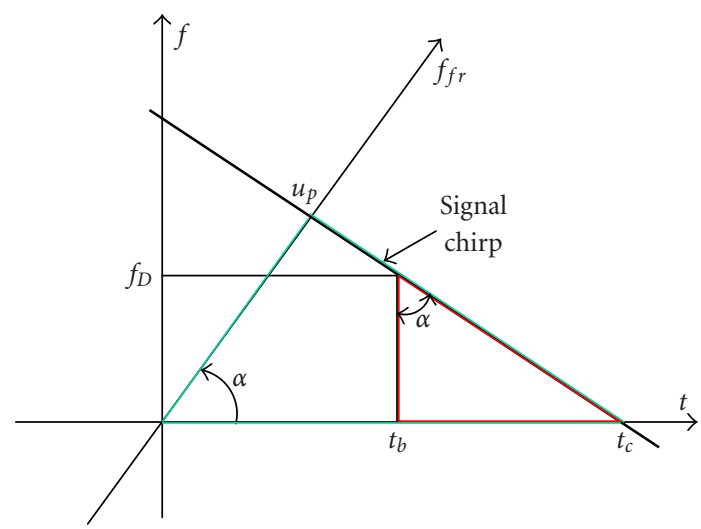

Figure 4: Derivation of $t_{b}$ from frequency-time geometry of target parameters.

The new approach permits parameter estimation of $v_{y}, v_{x}$, and $x_{b}=v_{a} t_{b}$ in the fractional Fourier domain using (11), (15), and (18) directly from range-compressed raw data without actually forming an SAR image.

\subsection{Nonnegligible along-track velocity}

When examining target track (or range) histories, one observes that they have breaks and missing portions (see Figure 8), during which times the targets appear invisible to the radar. As a result, the centers of the tracks may not always correspond to the "broadside times" of these targets. As can be seen in Figure 8, most tracks are not centered at their broadside times (i.e., on the highway). Thus, one cannot determine the target broadside time $t_{b}$ from the track history. But more importantly (as will be shown below), this time offset $\delta t$ of the target track center from $t_{b}$, together with a nonnegligible $v_{x}$ (with respect to $v_{a}$ ), causes the estimate of the target interferometric phase $\varphi_{\text {ATI }}$ to deviate from its "ideal" value.

If the phase of the signal received at channel 1 is

$$
\varphi_{1}(t)=-2 k R(t)=-2 k\left[R_{b}+v_{y} \gamma\left(t-t_{b}\right)+\frac{v_{\mathrm{rel}}^{2}}{2 R_{b}}\left(t-t_{b}\right)^{2}\right],
$$

then the phase of the signal at channel 2 in far-field approximation is

$$
\varphi_{2}(t)=\varphi_{1}(t)-k \mu(t) d
$$

where

$$
\mu(t) \approx \frac{v_{x}-v_{a}}{R_{b}}\left(t-t_{b}\right)
$$

is Taylor series expansion of the direction cosine about broadside time $t_{b}$. To coregister the two channels, the signal received at channel 2 is forward-time-shifted by $t=d / 2 v_{a}$ to 
yield a phase:

$$
\begin{aligned}
\varphi_{2}\left(t+\frac{d}{2 v_{a}}\right)= & -2 k R_{b}-2 k \gamma v_{y}\left(t-t_{b}\right)-k \gamma v_{y} \frac{d}{v_{a}} \\
& -k \frac{v_{\mathrm{rel}}^{2}}{R_{b}}\left(t-t_{b}\right)^{2}-\frac{k d}{R_{b}} v_{e}\left(t-t_{b}\right) \\
= & \varphi_{1}(t)-k \gamma v_{y} \frac{d}{v_{a}}-\frac{k d}{R_{b}} v_{e}\left(t-t_{b}\right),
\end{aligned}
$$

where

$$
\begin{aligned}
v_{e} & =\frac{\left(v_{x}-v_{a}\right)^{2}+\left(1-\gamma^{2}\right) v_{y}^{2}}{v_{a}}+\left(v_{x}-v_{a}\right) \\
& =\frac{v_{\mathrm{rel}}^{2}}{v_{a}}+\left(v_{x}-v_{a}\right) .
\end{aligned}
$$

If the signal track is of length $T$ and is not centered at $t_{b}$ (but at $t_{b}+\delta t$ ), then coregistered signals at apertures 1 and 2 can be written, respectively, as

$$
\begin{aligned}
& s_{1}=\operatorname{rect}\left[\frac{t-t_{b}-\delta t}{T}\right] e^{j \varphi_{1}(t)}, \\
& s_{2}=\operatorname{rect}\left[\frac{t-t_{b}-\delta t}{T}\right] e^{j \varphi_{2}\left(t+d / 2 v_{a}\right)} .
\end{aligned}
$$

Substituting (28) into (6) and integrating to maximize the target signal in fractional Fourier domain, one obtains

$$
\begin{aligned}
F_{\alpha 1}(u) & =e^{j \epsilon_{1}} \int_{-\infty}^{+\infty} \operatorname{rect}\left[\frac{t-t_{b}-\delta t}{T}\right] e^{j\left(\xi_{1} t+v_{1} t^{2}\right)} d t \\
& =e^{j \epsilon_{1}} \int_{t_{b}+\delta t-T / 2}^{t_{b}+\delta t+T / 2} e^{j \xi_{1} t} d t \\
& =e^{j\left[\epsilon_{1}+\xi_{1}\left(t_{b}+\delta t\right)\right]} T \operatorname{sinc}\left(\frac{\xi_{1} T}{2 \pi}\right),
\end{aligned}
$$

where

$$
\begin{gathered}
\epsilon_{1}=-2 k R_{b}+2 k \gamma v_{y} t_{b}-\frac{k v_{\mathrm{rel}}^{2} t_{b}^{2}}{R_{b}}+2 \pi a u^{2}, \\
\xi_{1}=-2 k \gamma v_{y}+\frac{2 k v_{\mathrm{rel}}^{2} t_{b}}{R_{b}}-4 \pi a b u, \\
v_{1}=-\frac{k v_{\mathrm{rel}}^{2}}{R_{b}}+2 \pi a=0 .
\end{gathered}
$$

Again, note that the target signal is maximized in the fractional Fourier domain when $\nu_{1}$ is equal to zero, which yields a "sinc" function in (30) and the optimum fractional angle $\alpha$ $(a=\cot \alpha / 2)$ :

$$
\alpha=\operatorname{acot}\left(\frac{k v_{\mathrm{rel}}^{2}}{\pi R_{b}}\right)
$$

Similarly, the optimum fractional Fourier transform of the coregistered signal received at channel 2 can be shown to

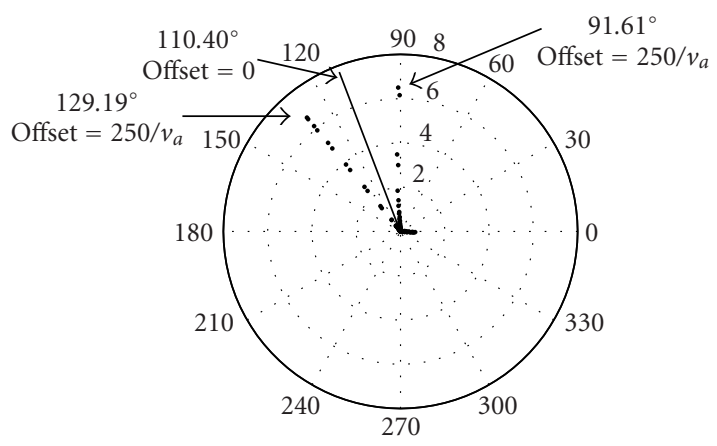

FIGURE 5: A simulated target's interferometric phases with significant center offset $\delta t$ in its track history. The target has velocity components $v_{x}=-25 \mathrm{~m} / \mathrm{s}$ and $v_{y}=5 \mathrm{~m} / \mathrm{s}$. The "ideal" phase with $\delta t=0$ is shown as solid line. The phase deviation from the ideal value is symmetric with respect to $\delta t=0$.

be

$$
F_{\alpha 2}(u)=e^{j\left[\epsilon_{2}+\xi_{2}\left(t_{b}+\delta t\right)\right]} T \operatorname{sinc}\left(\frac{\xi_{2} T}{2 \pi}\right)
$$

where

$$
\begin{gathered}
\epsilon_{2}=\epsilon_{1}-\frac{k \gamma v_{y} d}{v_{a}}+\frac{k t_{b} d v_{e}}{R_{b}}, \\
\xi_{2}=\xi_{1}-\frac{k d v_{e}}{R_{b}} .
\end{gathered}
$$

Therefore, the differential phase of interferogram $\left(\varphi_{\text {ATI }}=\right.$ $\left.\arg \left[F_{\alpha 1}(u) F_{\alpha 2}(u)^{*}\right]\right)$ evaluated at optimum fractional Fourier angle $\alpha$ can be shown to be equal to

$$
\varphi_{\mathrm{ATI}}=\frac{k d v_{y} \gamma}{v_{a}}+\frac{k d v_{e}}{R_{b}} \delta t
$$

which indicates that $\varphi_{\text {ATI }}$ is also sensitive to $v_{x}$ if the target track is not centered at $t_{b}$. Its sensitivity is dependent on the degree of offset $\delta t$ and the target-platform velocity difference $\left(v_{x}-v_{a}\right)$ in the along-track direction.

Figure 5 shows the ATI phase of a simulated moving target with its track history centered at $t=t_{b}+\delta t$. The "ideal" phase (i.e., when $\delta t=0$ ) is shown as solid line. The target moves at a constant velocity $\left(v_{x}=-25 \mathrm{~m} / \mathrm{s}\right.$ and $\left.v_{y}=5 \mathrm{~m} / \mathrm{s}\right)$ in a flat earth geometry as depicted in Figure 1. The dominant term in $v_{e}$ in (35) is along-track velocity $v_{x}$. Therefore, large $\delta t$ and $v_{x}$ values lead to significant deviation of ATI phase from the ideal value. One also notes that the phase deviation is symmetric with respect to $\delta t=0$.

In more general cases, therefore, $\delta t$ cannot be ignored and the estimation procedure proposed in Section 3.2 cannot be applied to obtain velocity and position estimates, if $v_{x}$ is non-negligible with respect to the platform velocity $v_{a}$. In order to resolve this, an additional independent equation is needed. This can be obtained by making use of "angleof-arrival" information. When unregistered signals from two 


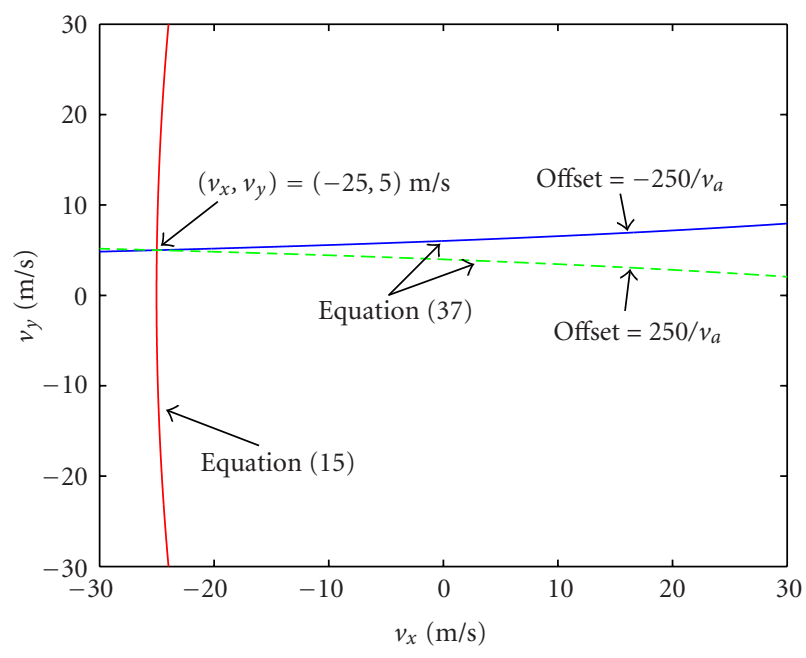

FIgURE 6: A plot of the simulated target's $v_{y}$ versus $v_{x}$ computed using (37) and (15). The new method takes into account the effect of offset $\delta t$ on ATI phase and yields correct parameter estimates.

identical channels are combined to form an interferogram, its differential phase (or angle) provides angle-of-arrival information of a target, instead of its velocity information as in the coregistered signal case. When the target is at broadside of the antenna beam, the distances between the target and the two receiving subapertures should be identical and, therefore, the phases of the two signals received at two channels should also be identical, which leads to a zero ATI phase. However, when $\delta t \neq 0$, the ATI phase computed from the two unregistered signals is no longer zero but can be shown to be

$$
\varphi_{\mathrm{ATI}_{u}}=\frac{k\left(v_{x}-v_{a}\right) d}{R_{b}} \delta t
$$

which is a function of both $v_{x}$ and $\delta t$. Substituting (36) into (35) to eliminate unknown parameter $\delta t$ and solving for $v_{y}$ result in

$$
v_{y}=\frac{v_{a}}{k \gamma d}\left\{\varphi_{\mathrm{ATI}}-\varphi_{\mathrm{ATI}_{u}}\left[\frac{R_{b} \lambda f_{s}^{2} \cot \alpha}{2 N v_{a}\left(v_{x}-v_{a}\right)}+1\right]\right\},
$$

which has only two unknowns, $v_{x}$ and $v_{y}$. Target velocity components can, therefore, be obtained from (37) and (15), and target azimuth position from (17). The revised parameter estimation procedure is applied to the above simulated target and its velocity components are estimated and plotted in Figure 6. Using the revised approach, the target parameters are now correctly estimated.

\subsection{Comparison to matched-filter approach}

Some of the advantages of the proposed FrFT approach become apparent when comparing with the matched-filter
(MF) method (i.e., via SAR azimuth compression). If one assumes a matched filter that matches exactly to the Doppler rate or $v_{\text {rel }}$ (i.e., the second coefficient in the Taylor expansion) of a constantly moving target but does not match correctly to its Doppler shift or $v_{y}$ (i.e., the first coefficient in the Taylor expansion), then the reference function for azimuth compression can be written as

$$
h(t)=\operatorname{rect}\left[\frac{t}{T_{r}}\right] \exp \left\{-j\left[2 k\left(v_{y}^{r} t+\frac{1}{2 R_{b}} v_{\text {rel }}^{2} t^{2}\right)\right]\right\},
$$

where $v_{y}^{r}$ and $T_{r}$ in (38) are not necessarily equal to $v_{y}$ and $T$, respectively, in (28) and (29). The resulting ATI phase obtained by solving

$$
\varphi_{\mathrm{ATI}_{\mathrm{mf}}}(t)=\arg \left[s_{1}(t) * h^{*}(-t)\right]\left[s_{2}(t) * h^{*}(-t)\right]^{*}
$$

can be shown to be equal [13] to

$$
\varphi_{\mathrm{ATI}_{\mathrm{mf}}}=\frac{k v_{y} \gamma d}{v_{a}}-\frac{k \gamma d v_{e}}{2 v_{\mathrm{rel}}^{2}}\left(v_{y}-v_{y}^{r}\right)+\frac{k d v_{e}}{R_{b}}\left[\frac{\delta t}{2} \mp \frac{T_{r}-T}{4}\right],
$$

where “ $\mp$ ” corresponds to cases where $\left(t_{c}-t_{b}-\delta t\right)>0$ and $\left(t_{c}-t_{b}-\delta t\right)<0$, respectively, and $t_{c}$ is the azimuthshifted-time position of target, which is also the time of closest approach if the MF is matched to the target's Doppler rate. In order to correctly estimate the target ATI phase, which is a key parameter for target velocity estimation, the MF approach requires matching to $v_{\text {rel }}$ and $v_{y}$ simultaneously. This is more difficult to accomplish than matching only to $v_{\text {rel }}$ as in the FrFT case. The expression (40) also indicates that if one does not correctly choose a $v_{y}^{r}$ for the reference function (i.e., $v_{y}^{r} \neq v_{y}$ ), the azimuth processing actually leads to a biased or erroneous ATI phase because of its " $v_{y}-v_{y}^{r}$ " dependence. A simulation run of a moving target with $v_{x}=-25 \mathrm{~m} / \mathrm{s}$ and $v_{y}=5 \mathrm{~m} / \mathrm{s}$ in background Gaussian clutter and using a matched filter perfectly matched to $v_{\text {rel }}$ but not to $v_{y}\left(v_{y}^{r}=0\right)$, clearly shows that the target's ATI phase, computed via the MF approach, deviates strongly from the ideal phase value computed using (11), as shown as solid lines in Figure 7. The ideal ATI phase is $110.4^{\circ}$, but the SAR approach consistently yields biased results for various SCRs. The SCR is here defined to be the target's SCR before pulse compression. Even with sufficiently large SCR, the ATI phase is still biased at $101.2^{\circ}$, which is predicted and confirmed by (40). In addition to its dependence on $\delta t$ and " $v_{y}^{r}-v_{y}$," the MF method is also dependent on " $T_{r}-T$," which means that the choice of matched-filter length will also affect the resulting ATI phase value.

Also shown in the figure are the results computed using the FrFT method (shown as dots). Without a $t_{b}$ offset (i.e., $\delta t=0$ ), the technique yields the ideal ATI phase, except when the SCR is small and the ATI phase becomes severely contaminated by the interfering clutter. It is clear, therefore, that the FrFT approach is superior to the MF method because the FrFT approach requires only matching the target's Doppler rate, which is a relatively straight-forward and robust procedure. The MF, on the other hand, involves matching not only 


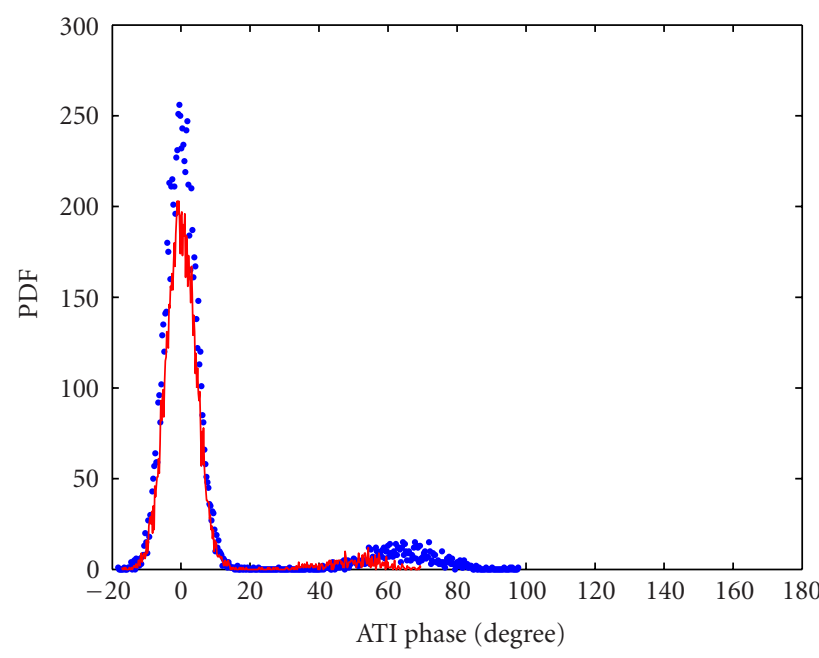

(a)

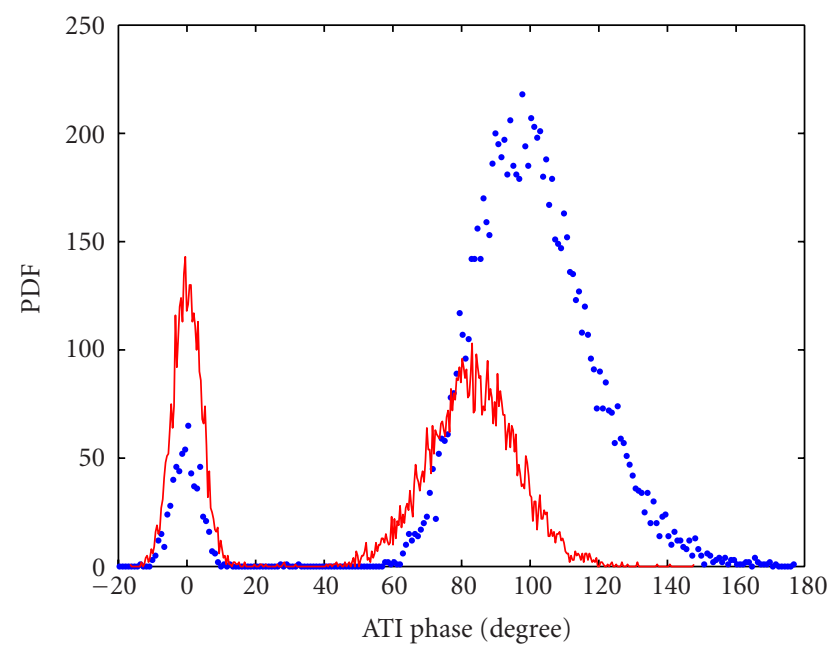

(c)

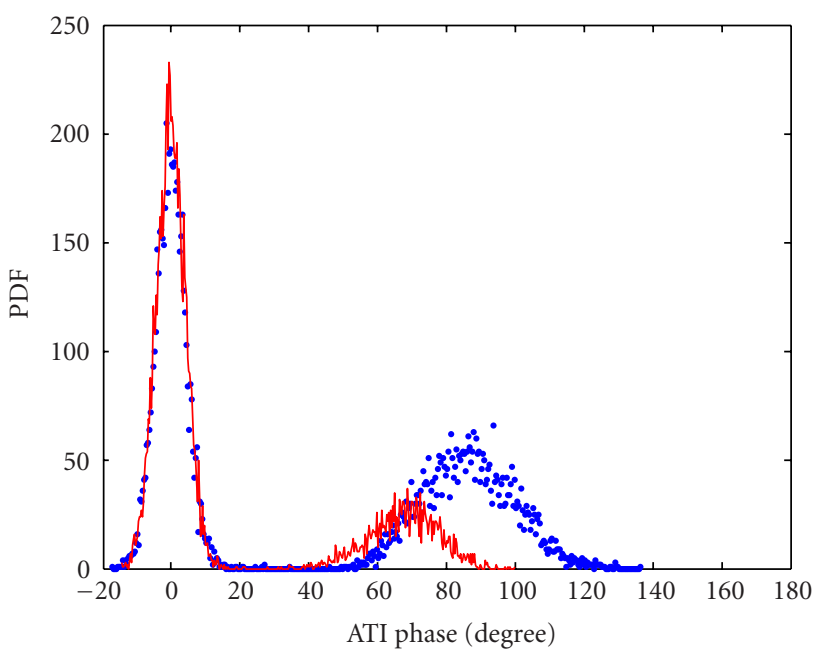

(b)

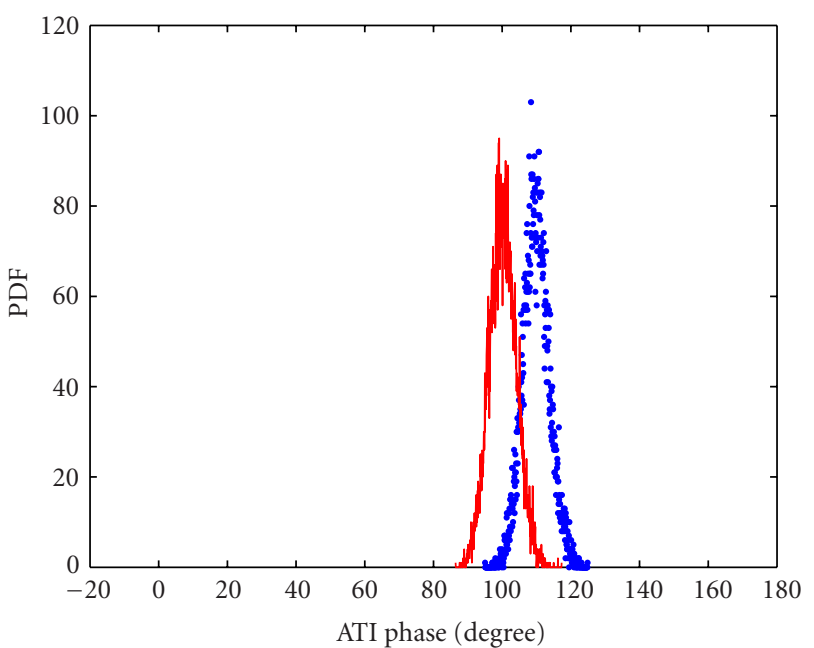

(d)

FIGURE 7: A simulated deterministic target with $v_{x}=-25 \mathrm{~m} / \mathrm{s}$ and $v_{y}=5 \mathrm{~m} / \mathrm{s}$ in a background Gaussian clutter, using a matched filter perfectly matched to $v_{\text {rel }}$ but not to $v_{y}\left(v_{y}^{\prime}=0\right)$. Assume $\delta t=0$. The ideal ATI phase is $110.4^{\circ}$. The FrFT and MF results are denoted by dots and solid lines, respectively. (a) SCR $=-24.4 \mathrm{~dB}$; (b) SCR $=-21.9 \mathrm{~dB}$; (c) SCR $=-20.0 \mathrm{~dB}$; and (d) SCR $=-6.0 \mathrm{~dB}$. The signal length is equal to the matched-filter length.

to the target's Doppler rate but also to its Doppler shift in order to avoid any bias, a process proven to be difficult and unreliable due to insufficient information.

\section{AIRBORNE EXPERIMENT}

As part of the preparatory work for the RADARSAT-2 MODEX, airborne experiments were conducted to acquire SAR-ATI data for typical RADARSAT-2 resolutions and angles of incidence. The dataset used in this study was obtained at Canadian Forces Base (CFB), Petawawa, on November 5, 2000, by the Environment Canada CV 580 C-band SAR configured in its along-track interferometer mode [14]. The study focuses on targets of opportunity (TOOs) on Highway 17 , which runs through the experimental site. The highway was monitored by two video cameras $600 \mathrm{~m}$ apart set up along a stretch of the highway to measure TOO speeds. The highway has a speed limit of $90 \mathrm{~km} / \mathrm{h}$, but most TOOs monitored were $10-20 \mathrm{~km} / \mathrm{h}$ over the limit. The video cameras monitored over 47 vehicles during the data acquisition period, and their measured ground speeds $v_{g}$ varied from $83 \mathrm{~km} / \mathrm{h}$ to $120 \mathrm{~km} / \mathrm{h}$.

Targets were detected in the range-compressed raw-data domain via a combination of the DPCA technique [9], which 


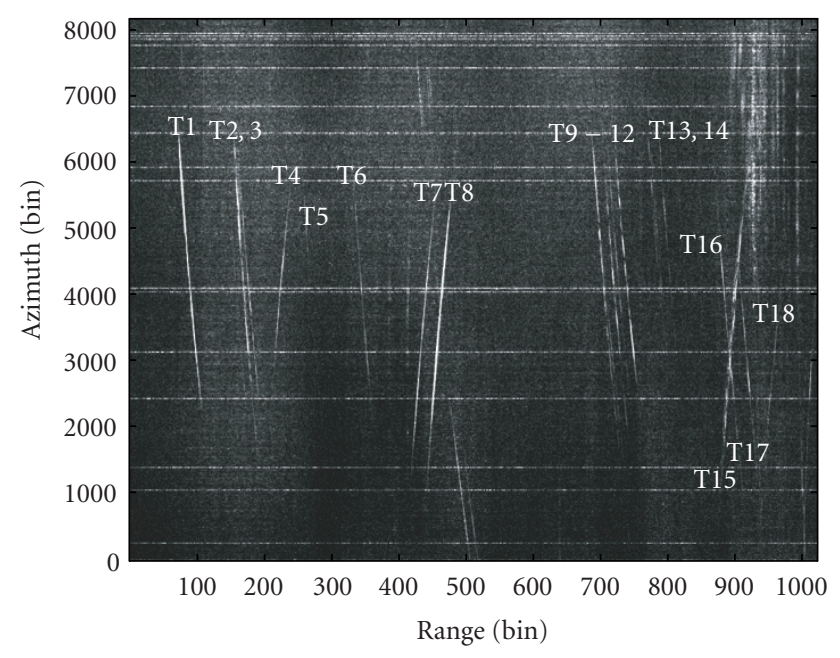

FIGURE 8: Range-compressed raw-data DPCA output. The horizontal lines are due to motion compensation artifacts.

subtracts the aft channel from the coregistered fore channel to suppress the background clutter, and the raw-data target detection algorithm developed by Gierull and Sikaneta $[13,15]$. The DPCA is first applied to the pair of fore and aft channel range-compressed signal data to obtain a plot of target track histories as shown in Figure 8. Since the targets are azimuthally uncompressed, their energies are spread over the slow time. Since these targets are moving on Highway 17 along the across-track direction, their track histories are slanted in range as seen in Figure 8, indicating range walk. Uncancelled bright stationary targets also show up in the image, as seen in the upper right-hand corner of Figure 8 (where a small town is located), but their tracks are seen as almost vertical (actually they are hyperbolas symmetric to the across-track direction). In order to estimate the target parameters, Gierull and Sikaneta's automatic detection algorithm [15] is first applied to extract their signal tracks. Eighteen moving targets are detected and analyzed.

Figure 9 shows the polar plots of three (T4, T9, and T10) of the eighteen detected targets' interferograms. Figures 9a, $9 \mathrm{~b}$, and $9 \mathrm{c}$ are interferograms obtained from the regular SAR-ATI processing (i.e., from the fore and aft SAR images processed by a stationary terrain matched filter). Interferograms for the same targets obtained from the FrFT processing are shown in Figures 9d, 9e, and 9f. The signal points near the zero phase radial belong to stationary clutter. As can be seen, ATI phases obtained from the regular SAR processing are severely contaminated by the interfering clutter and their values are consistently lower than those obtained from the FrFT, which gives not only much cleaner and better defined phases but also correct values compared to their true phases. The "true" phase is here defined to be the $\varphi_{\text {ATI }}$ that gives the correct azimuth correction to bring a target back onto the road (or Highway 17).

The analysis results of all eighteen targets are summarized in Table 1, showing targets' interferometric phases $\varphi_{\text {ATI }}$ and their estimated ground velocities $v_{g}$. The velocities and azimuth shifts of 10 targets are correctly estimated by the FrFT approach compared to only three by the regular SAR approach.

Since all targets are either moving almost directly towards or directly away from the radar, their radial velocities $v_{r}$ are both high and ambiguous due to phase wrapping. The velocity ambiguity can be resolved by using the track history of each target, as the one shown in Figure 10 for target T1. By converting azimuth samples to azimuth time (i.e., multiplying the number of azimuth samples by the pulse repetition frequency) and range samples to range distance (i.e., multiplying the number of range samples by $c / f_{\mathrm{A} / \mathrm{D}}$, where $c$ is the speed of light and $f_{\mathrm{A} / \mathrm{D}}$ is $\mathrm{A} / \mathrm{D}$ sampling frequency), one obtains an average $v_{y}$ of $+109 \mathrm{~km} / \mathrm{h}$ for target T1. Therefore, the ATI phase is expected to wrap around $2 \pi$, which occurs at $v_{r}=13.15 \mathrm{~m} / \mathrm{s}(47.33 \mathrm{~km} / \mathrm{h})$. In fact, the measured ATI phases for all targets under consideration must be unwrapped to yield target speeds that fall within the monitored speed range ( 83 to $120 \mathrm{~km} / \mathrm{h}$ ). For instance, T1's $-132^{\circ}$ phase is in fact $+588^{\circ}$, which yields a $v_{r}=+77.3 \mathrm{~km} / \mathrm{h}$, or a ground velocity of $+106.2 \mathrm{~km} / \mathrm{h}$ after taking into account the imaging geometry.

Figure 11 plots the targets' azimuthally-corrected positions estimated from the proposed FrFT approach. The circles represent targets in their azimuth-shifted positions and the squares are their corrected locations. As can be seen, ten out of eighteen targets are correctly positioned on Highway 17. The other targets are not because their SCRs remain small $(\lesssim-3 \mathrm{~dB})$ even after the FrFT processing.

The estimation procedure proposed in Section 3.1 yields good positional estimates for the 18 targets examined, even though the majority of the target tracks may not be centered at broadside. This is because most targets examined are moving mainly toward or away from radar with a relatively small $v_{x}$ component, usually in the range of 2 to $5 \mathrm{~m} / \mathrm{s}$. This is especially true for targets T1 through T12. Therefore, track center offsets do not significantly affect the $t_{b}$ estimates.

\section{CONCLUSIONS}

Moving target parameter estimation using FrFT methods, in combination with the ATI, is here demonstrated for the first time. This new proposed approach is compared to the MF method and is shown to be less biased and more robust. In particular, the ATI phase computed via FrFT is not dependent on the target's across-track velocity component $\left(v_{y}\right)$ or its Doppler shift, which is difficult to measure experimentally due to insufficient degrees of freedom. The phase (FrFT) depends only on target's Doppler rate, which is shown to be measurable experimentally with a high degree of robustness. The ATI phase via MF, on the other hand, is dependent not only on the target's Doppler rate but also on $v_{y}$. Moreover, the selection of MF length directly affects the ATI phase estimate. All these make the MF a less desirable method than the proposed FrFT approach. The FrFT method also allows the estimation of target's true azimuth position $x_{b}$ (or the so-called "broadside" position) directly from its measured 


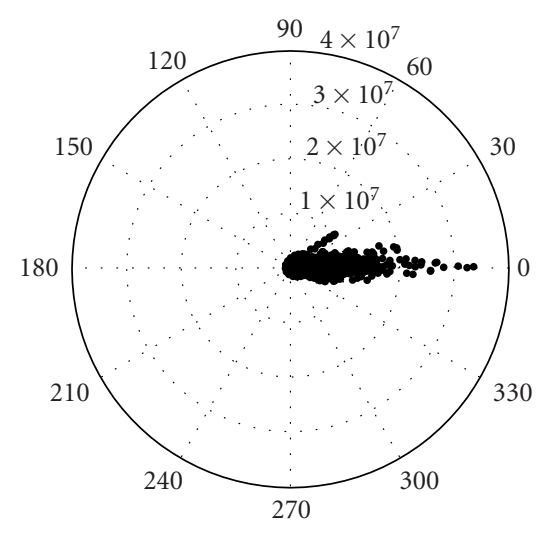

(a)

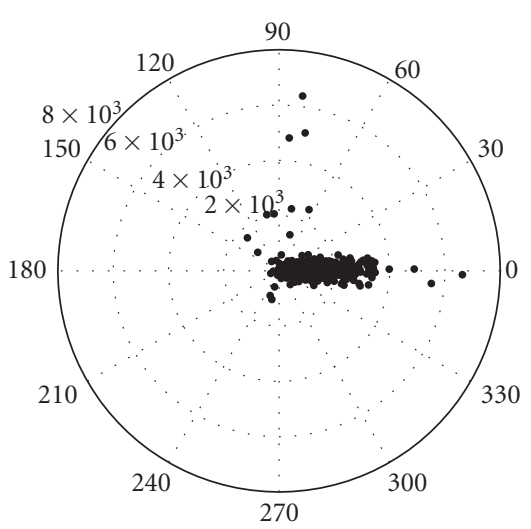

(d)

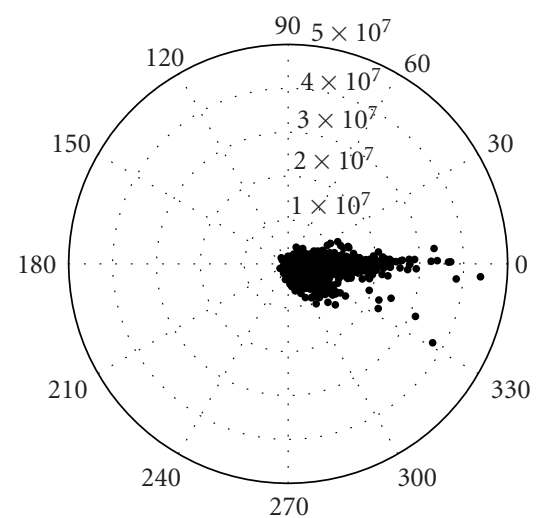

(b)

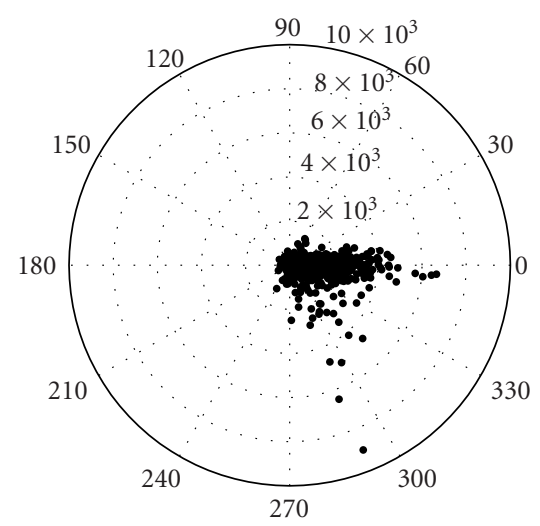

(e)

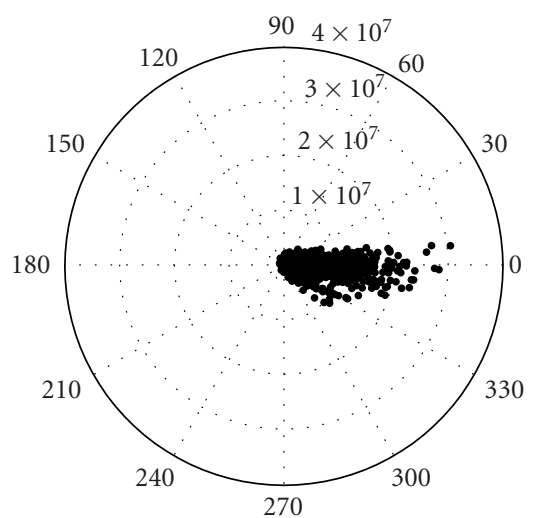

(c)

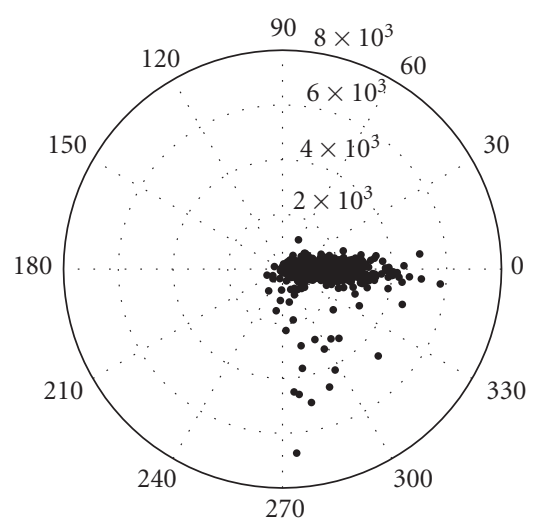

(f)

FIgUre 9: (a), (b), and (c) are clutter-corrupted ATI signals for targets T4, T9, and T10, respectively; (d), (e), and (f) are the same targets' ATI signals after the FrFT filtering or focussing.

TABLE 1: Estimated interferometric phase $\varphi_{\text {ATI }}$ and ground velocity $v_{g}$ of eighteen detected targets.

\begin{tabular}{c|ccc}
\hline Target ID & SAR $\varphi_{\text {ATI }}($ degree $) / v_{g}(\mathrm{~km} / \mathrm{h})$ & FrFT $\varphi_{\text {ATI }}($ degree $) / v_{g}(\mathrm{~km} / \mathrm{h})$ & “True” $\varphi_{\text {ATI }}($ degree $) / v_{g}(\mathrm{~km} / \mathrm{h})$ \\
\hline T1 & $-142 / 104.5$ & $-132.7 / 106.2$ & $-131 / 106.5$ \\
T2 & $-42 / 118.9$ & $-42.8 / 118.7$ & $-43 / 118.7$ \\
T3 & $0 / 0$ & $-87 / 110.7$ & $-157.7 / 98.4$ \\
T4 & $+32 / 118.7$ & $+80 / 110.4$ & $+79 / 110.6$ \\
T5 & & Target too weak for estimation $)$ & $-71.3 / 108.5$ \\
T6 & $-60 / 110.4$ & $-71 / 108.5$ & $+100 / 101.8$ \\
T7 & $+106 / 100.8$ & $+103 / 101.3$ & $+85 / 103.7$ \\
T8 & $+88 / 103.2$ & $+85.4 / 103.6$ & $-63.5 / 104.7$ \\
T9 & $-26 / 110.7$ & $-65 / 104.5$ & $-84 / 101.3$ \\
T10 & $-30 / 109.9$ & $-85 / 101.1$ & $-104.5 / 98.0$ \\
T11 & $-5 / 113.8$ & $-70 / 103.4$ & $-137 / 92.7$ \\
T12 & $0 / 0$ & $-135 / 93.0$ & $-83 / 100.6$ \\
T13 & $-60 / 104.2$ & $-10 / 112.1$ & $-86.5 / 99.8$ \\
T14 & $-47 / 106.0$ & $-48 / 105.9$ & $+44 / 122.2$ \\
T15 & $+30 / 124.7$ & $+45 / 122.0$ & $-150 / 103.1$ \\
T16 & $-32 / 124.4$ & $-130 / 106.7$ & $-153.5 / 102.2$ \\
T17 & $0 / 0$ & $-82 / 115.0$ & $+98.5 / 111.0$ \\
T18 & $0 / 0$ & $+44 / 120.8$ & \\
\hline
\end{tabular}




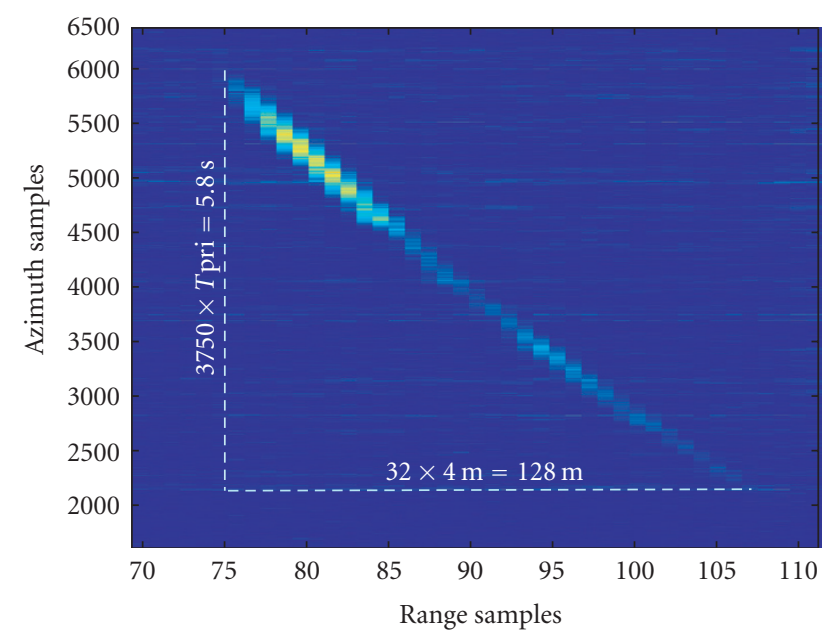

Figure 10: Track history of target T1 extracted from Figure 8.

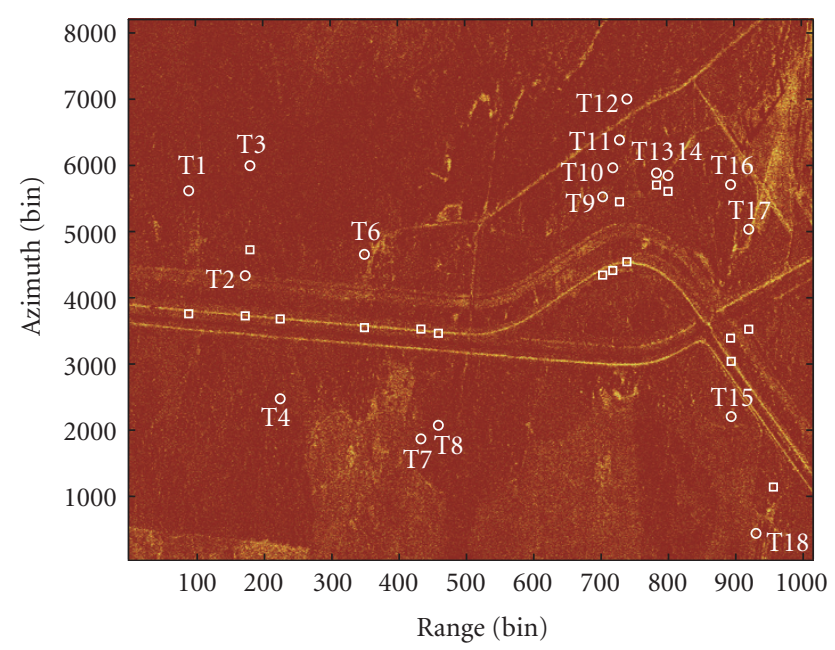

FIgURE 11: Detected moving targets in the SAR image context. The circles represent target azimuth-shifted positions and the squares are azimuth-corrected positions.

position $u_{p}$ in the fractional Fourier domain without actually forming an SAR image or knowing the position of "closest approach" $t_{c}$.

\section{REFERENCES}

[1] C. E. Livingstone, "The addition of MTI modes to commercial SAR satellites," in Proc. 10th CASI Conference on Astronautics, pp. 267-275, Ottawa, Canada, October 1998.

[2] E. F. Stockburger and D. N. Held, "Interferometric moving ground target imaging," in Proc. Record of the IEEE 1995 International Radar Conference, pp. 438-443, Alexandria, Va, USA, May 1995.

[3] E. Yadin, "A performance evaluation model for a two port interferometer SAR-MTI," in Proc. IEEE 1996 National Radar Conference, pp. 261-266, Ann Arbor, Mich, USA, May 1996.
[4] R. K. Raney, "Synthetic aperture imaging radar and moving targets," IEEE Trans. Aerosp. Electron. Syst., vol. 7, no. 3, pp. 499-505, 1971.

[5] E. Yadin, "Evaluation of noise and clutter induced relocation errors in SAR MTI," in Proc. Record of The IEEE 1995 International Radar Conference, pp. 650-655, Alexandria, Va, USA, May 1995.

[6] B. Boashash, "Heuristic formulation of time-frequency distributions," in Time Frequency Signal Analysis and Processing, pp. 38-41, Elsevier, Amsterdam, The Netherlands, 2003.

[7] L. B. Almeida, "The fractional Fourier transform and timefrequency representations," IEEE Trans. Signal Processing, vol. 42, no. 11, pp. 3084-3091, 1994.

[8] A. I. Zayed, "On the relationship between the Fourier and fractional Fourier transforms," IEEE Signal Processing Lett., vol. 3, no. 12, pp. 310-311, 1996.

[9] F. R. Dickey Jr. and M. M. Santa, "Final report on anticlutter techniques," General Electric Company Rep. R65EMH37, Fairfield, Conn, USA, 1953.

[10] S. Chiu, "Clutter effects on ground moving target velocity estimation with SAR along-track interferometry," in Proc. International Geoscience and Remote Sensing Symposium (IGARSS '03), pp. 1314-1319, Toulouse, France, July 2003.

[11] J. J. Sharma and M. J. Collins, "Focusing accelerating ground moving targets in SAR imagery," in Proc. 5th European Conference on Synthetic Aperture Radar (EUSAR '04), pp. 841-844, Ulm, Germany, May 2004.

[12] R. M. Goldstein and H. A. Zebker, "Interferometric radar measurement of ocean surface currents," Nature, vol. 328, no. 20, pp. 707-709, 1987.

[13] C. H. Gierull and I. C. Sikaneta, "Ground moving target parameter estimation for two-channel SAR," in Proc. 5th European Conference on Synthetic Aperture Radar (EUSAR '04), pp. 799-802, Ulm, Germany, May 2004.

[14] C. E. Livingstone, I. C. Sikaneta, C. H. Gierull, et al., "An airborne synthetic aperture radar (SAR) experiment to support RADARSAT-2 ground moving target indication (GMTI)," The Canadian Journal of Remote Sensing, vol. 28, no. 6, pp. 794-813, 2002.

[15] C. H. Gierull and I. C. Sikaneta, "Raw data based two-aperture SAR ground moving target indication," in Proc. International Geoscience and Remote Sensing Symposium (IGARSS '03), vol. 2, pp. 1032-1034, Toulouse, France, July 2003.

Shen Chiu received his B.A.S. and Ph.D. degrees in engineering physics from the University of British Columbia, in 1987, and the University of Toronto, in 1993, respectively. From 1994 to 1997, he worked as a Research Associate at the Université du Quebec on chemical vapor deposition of large bandgap thin films. From 1997 to early 1998, he was with New York State Center for Advanced Thin Film Technology, where he worked on

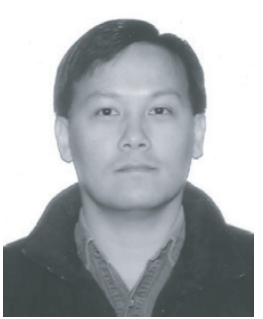
advanced copper metallization for ULSI and on electroluminescent flatpanel displays based on solid-state thin film technologies. Presently, he is a Defence Scientist at Defence R\&D Canada, Ottawa, where he has developed microfabrication techniques to manufacture high-efficiency quantum well infrared photodetectors (QWIP). Since 1999, he has been a member of the RADARSAT-2 GMTI research team actively developing the RADARSAT-2 GMTI processor. 